\title{
Engineering tendon and ligament tissues: present developments towards successful clinical products
}

\author{
Márcia T. Rodrigues, Rui L. Reis and Manuela E. Gomes* \\ 3Bs Research Group - Biomaterials, Biodegradables and Biomimetics, University of Minho, Headquarters of the European Institute of \\ Excellence on Tissue Engineering and Regenerative Medicine, AvePark, 4806-909 Taipas, Guimarães, Portugal
}

\begin{abstract}
Musculoskeletal diseases are one of the leading causes of disability worldwide. Among them, tendon and ligament injuries represent an important aspect to consider in both athletes and active working people. Tendon and ligament damage is an important cause of joint instability, and progresses into early onset of osteoarthritis, pain, disability and eventually the need for joint replacement surgery. The social and economical burden associated with these medical conditions presents a compelling argument for greater understanding and expanding research on this issue. The particular physiology of tendons and ligaments (avascular, hypocellular and overall structural mechanical features) makes it difficult for currently available treatments to reach a complete and long-term functional repair of the damaged tissue, especially when complete tear occurs. Despite the effort, the treatment modalities for tendon and ligament are suboptimal, which have led to the development of alternative therapies, such as the delivery of growth factors, development of engineered scaffolds or the application of stem cells, which have been approached in this review. Copyright @ 2012 John Wiley \& Sons, Ltd.
\end{abstract}

Received 22 November 2011; Accepted 24 November 2011

Keywords tendon/ligament; tendon/ligament repair modalities; tendon/ligament tissue engineering strategies

\section{Background}

Tendon and ligament tissues have a pivotal mechanical role in joint stability, due to their poor natural healing capacity. Injuries in these tissues severely affect the joint functionality, which can ultimately result in the progression of degenerative diseases (Fleming et al., 2005). Tendon and ligament damage is frequent and is responsible for substantial morbidity in athletes, working and elder population, thus representing a significant cost to the community in social, economic and health terms. Currently used therapies are mainly limited to pain control and/or tissue replacement, without fully restoring tissue functionality.

Tissue-engineering strategies, such as growth and differentiation factor delivery, development of engineered

\footnotetext{
*Correspondence to: M. E. Gomes, 3Bs Research Group - Biomaterials, Biodegradables and Biomimetics, University of Minho, Headquarters of the European Institute of Excellence on Tissue Engineering and Regenerative Medicine, AvePark, 4806-909 Taipas, Guimarães, Portugal.

E-mail:megomes@dep.uminho.pt
}

scaffolds and/or the integration of stem cells, are generating potential areas for additional prospective investigation in tendon or ligament regeneration. Nevertheless, significant challenges remain to accomplish a complete and functional tendon and ligament repair that will lead to a clinically effective and commercially successful application.

\section{Tendon and ligament tissues: affinities and dissimilarities}

Tendons and ligaments are similar dense fibrous connective tissues that connect skeletal muscle to the skeletal elements (bone) and bone to bone (Frank, 2004), respectively. Both tissues are also characterized by the presence of fibroblasts/fibrocytes (ligament) or tenoblasts/tenocytes (tendon) and an abundant extracellular matrix (ECM), mainly composed of collagen I (Gelse et al., 2003), resulting in a dense and hypocellular structure. The tendon matrix is maintained by the resident tenocytes, in a continuous process of matrix remodelling. Although the rate of ECM turnover varies at different sites, it is strongly influenced by physical activity (Kjaer 
et al., 2005; Reeves, 2006). In pathological conditions, other cell types, such as inflammatory cells, macrophages and non-resident fibroblasts, might be present in these tissues.

Tenoblasts vary in shape and size, ranging from 20 to $70 \mu \mathrm{m}$ in length. When tenoblasts mature into tenocytes, they became very elongated, with a width in the range $80-300 \mu \mathrm{m}$. Tenocytes are active in generation and synthesis of collagen and all other components of the ECM. The low metabolic rate is essential to carry loads and maintain tension of long periods, reducing the risk of ischaemia and subsequent necrosis (Sharma and Maffulli, 2005).

Ligaments contain about two-thirds water by weight. The remaining one-third consists mainly of collagen, elastin, glycosaminoglycans (GAGs), cells and other biochemical molecules ( $\mathrm{Ng}, 2002)$. Ligaments contain more protein, less total collagen and greater proportions of type III collagen and GAGs than tendons ( $\mathrm{Ng}, 2002)$. In general, ligaments are more active than tendons.

Ligaments and tendons share physiological and constituent features, with a similar hierarchical structure that affects their mechanical behaviour. Thus, in most cases, the regenerative approach to follow is similar, and sometimes the terms 'tendon' and 'ligament' are intermingled in the literature. Nevertheless, these structures have different functional roles in the human body.

Tendons function as structural components and play a mechanical role in linking and transmitting forces generated by muscle to bone, resulting in joint movement. Moreover, tendons also act as springs, modulating forces during locomotion and providing additional stability and a significant degree of resistance to external forces, which also allows tendons to store and recover energy at high efficiency.

The major function of ligaments is mechanical, as they passively stabilize joints and help in guiding those joints through their normal range of motion when a tensile load is applied (Frank, 2004). Capsular ligaments act as mechanical reinforcements, while extra-capsular ligaments join together and provide joint stability.

During development, maturation and ageing, alterations in composition, structure and biomechanics occur in tendons and ligaments (Frank, 2004; Reeves, 2006), reducing the optimal functioning of the locomotive system. Furthermore, mature cells are also affected by a reduced metabolic rate and vascular supply. Although the normal mechanical function depends on precise alignment of collagen fibrils, proteoglycans (PGs) regulate collagen fibrillogenesis and therefore indirectly modulate tissue function (Yoon and Halper, 2005). Furthermore, PGs play a major role in structural and biochemical adaptation to changes in loading, and are thus responsible for maintaining proper biomechanical function (Yoon and Halper, 2005). Continued loss of PGs and other matrix components appears to activate loss of collagen. Consequently, the load absorption and redistribution properties are less efficient and render the tissue more prone to injury. Changes in PGs expression and metabolism/turnover have been associated with tendinopathy.

\section{Tendon and ligament injuries}

Tendon/ligament injuries may be caused by trauma, tumour resection, atrophy or even shortening after tendon laceration. These are prevalent and debilitating lesions that affect the quality of life among populations worldwide and can be caused by intrinsic and/or extrinsic factors (Riley, 2004). Disease or body weight are intrinsic factors associated with tendon injuries, although some forms of injury may already be predisposed in patients, such as biomechanical irregularities (anatomical and/or functional misalignment, muscle imbalance) or genetic susceptibility for developing full-thickness tears (Carr et al., 2004). Extrinsic factors include environmental conditions, prescription drugs, nutrition and lifestyle (Maffulli et al., 2003). These factors are more common among people whose occupations or recreational athletic activities require repetitive motion of the shoulder, knee, elbow or ankle joints.

The increasing levels of activity, and thus of mechanical loading on tendon/ligament tissues, of the elder population, is also likely to contribute to these injuries. Ageing is involved in tendon/ligament injuries, influencing the composition and stability of the ECM. Nevertheless, studies performed on human patients are uncertain regarding determining age as a risk factor in tendon/ligament injuries (Couppe et al., 2009).

Patient gender is also a subject of debate. Although gender is not considered a risk factor, sex hormones strongly influence collagen synthesis and, as a result, tendon/ligament composition. Moreover, anatomical differences (e.g. tendon/ ligament size) between men and women may have implications in tendon/ligament injuries (Carroll et al., 2008).

Despite all the factors mentioned above, mechanical loading is the major factor for cellular mechanobiological responses that may lead to tendon physiological remodelling or pathological changes, such as tendinopathy. Tendinopathy is associated with sports and physical activity in active people over 25 years of age, and refers to the clinical conditions in and around tendons resulting from overload and overuse. It requires lengthy management, and patients often respond poorly to treatment. Frequent tendinopathies include tendinitis, which can be described as an inflammatory injury, and tendinosis, often associated with non-inflammatory chronic degeneration or weakening of the tendons, which may eventually lead to tendon rupture.

Changes in cellularity and in the remodelling activity of tendon ECM are associated with the onset of tendinopathies. The most frequently affected tendons are highly stressed tissues, often exposed to repeated strains including shear or compressive forces, and are relatively less vascularized (Riley, 2004). Patellar, Achilles and hand and foot flexor tendons are among the areas most affected by tendinopathies.

Patellar tendinopathy (PT) is a chronic painful degenerative condition that accounts for over 30\% of sportsrelated injuries (Sharma and Maffulli, 2006). Overuse of the patellar tendon can lead to pain, tenderness, 
functional deficit and disability in professional as well as in recreational athletes. Studies on the effectiveness of surgical treatment for PT remain inconclusive, due to the heterogeneity of surgical procedures and the limited clinical trials with control non-treated groups. Nevertheless, physiotherapy may be recommended to strengthen the muscles and tendons.

The Achilles tendon is the largest and the most powerful tendon in the body, enabling walking, running and jumping. The most common Achilles tendon injuries are Achilles tendinosis and Achilles tendon rupture, whose damage is a debilitating occurrence. The two main types of treatment are non-operative approaches and operative methods (Khan et al., 2005). Non-surgical procedures include rest, muscle strengthening, physical therapies for misalignment rectification, and non-steroidal anti-inflammatory drugs. Depending on the severity of the injury, recovery from an Achilles injury implies complete immobilization or, in the most severe cases, surgery.

The flexor tendons (FTs) in the hand and foot are highly complex and intricate. Related injuries often lead to significant morbidity for patients and are frequently associated with nerve and vascular injuries. Restoration of satisfactory digital function after FT lacerations remains one of the most challenging problems in hand surgery, as it requires the reestablishment of the continuity of the tendon fibres and of the gliding mechanism between the tendon and its surrounding structures (Beredjiklian, 2003).

All of the body ligaments are important to perform efficient physical movements. If these tissues are injured and are not effectively repaired, normal locomotion and joint structural integrity are compromised. The mechanisms of ligament injury are multifactorial and can be caused by contact or direct trauma, dynamic loading, repetitive overuse, structural vulnerability, poor flexibility, muscle imbalance or rapid growth (Curwin, 2005). Activityrelated injuries rarely involve damage to an isolated ligament but commonly result in a number of ligaments being injured, with an assortment of insults occurring in other associated tissues. The injury can happen with a single load that exceeds its maximum strain or from cumulative overload (repetitive sprains) with insufficient recovery time (Curwin, 2005).

Although ligaments can be composed of parallel or random fibres, ligament failure is associated with the degree of tearing of their collagen bundles. In micro-failure, ligaments undergo partial tearing or sprain, while great loads will result in complete ligament damage. Although minor ligament sprains may only cause annoyance and minimal function loss, these injuries can progress into severe ligament sprains, increasing functional loss. The most commonly and severely injured ligament is the anterior cruciate ligament (ACL), essential to knee function.

The anterior cruciate ligament (ACL) is the primary stabilizer of knee motion, acting as a guide rope during the screw-home mechanism of knee extension by preventing anterior translation of the tibia and hyperextension of the knee. The mechanical properties of the ACL, crucial to its function, are complex, showing variable tension among fibre bundles and variable modulus as a function of load level and viscoelastic effects (creep, relaxation and strain-rate sensitivity). The ACL is the most frequently injured knee ligament, especially in athletes, accounting for 200000 injuries and approximately 100000 reconstruction procedures reported annually in the USA alone (Gotlin and Huie, 2000; Majewski et al., 2006). Most patients are 20-29years of age but gender incidence is still a subject of debate (Majewski et al., 2006). Lateral rotational movements cause the ACL to strain or tear. Damage in ACL is usually accompanied by meniscus, medial cruciate ligament and knee cartilage tears. The most common procedure for repairing ACL injuries is surgery. However, ACL regeneration is poor and may be also affected by the little surrounding soft tissue available to promote extrinsic healing. Rupture of knee ligaments leads to instability with concomitant abnormal loading patterns. Over time, these abnormal loads can cause joint tissue degeneration, ultimately leading to the development of osteoarthritis (Fleming et al., 2005).

\section{Tendon/ligament healing}

\subsection{Natural healing mechanisms}

Regeneration of ligaments and tendons is a slow process and occurs from intrinsic or extrinsic healing or a combination of both. Although damaged tendon/ligament tends to heal, the repair is slow and inefficient after injury, never restoring the biological and biomechanical properties completely.

The intrinsic healing mechanism results from the local tenocytes/fibrocytes, while the extrinsic healing starts when fibroblasts and inflammatory cells from tendon sheath and surrounding soft tissues invade and proliferate to lay down a new collagen matrix. Intrinsic healing is dependent on resident cells proliferation, on an adequate blood supply and nutrition by surrounding fluids and on the lack of adhesion formation. The involvement of external cells in extrinsic healing also depends on the site of injury and vascular perfusion to the lesion site. Generally, exogenous fibroblasts predominate over resident cells, allowing the surrounding tissues to attach to the repair site, resulting in adhesion formation.

As in any other tissue injury, inflammation is the process by which tendons and ligaments heal upon damage, through three sequential stages: inflammation, repair and remodelling (Beredjiklian, 2003; Frank, 2004). The goal of this inflammatory-reparative cascade is to regenerate collagen, in particular, and the ECM in general, providing the connective tissues with strength and their characteristic ability to handle great strain forces.

In the inflammatory phase, which occurs immediately after the injury, and lasts from $24 \mathrm{~h}$ to 5 days, the inflammatory cells migrate to the wound site, phagocytose necrotic tissue and clot. These cells also release factors for recruiting fibroblasts to initiate collagen synthesis and deposition. 
A few days after the injury, the repair or fibroblastic phase begins. This phase, which lasts from 5 days to a few weeks, is characterized by proliferation or fibroplasia. Tendon/ligament fibroblasts synthesize abundant collagen and other ECM components, such as proteoglycans, and deposit them at the wound site to increase ligament and tendon strength.

The remodelling phase occurs between 6 weeks to 9 months after the original injury, when the organism attempts to restructure the lesion to a pre-injury state. This phase is characterized by decreased cellularity and decreased collagen and glycosaminoglycan synthesis. Cellular metabolism and vascularity also decline, and the repair tissue changes to fibrous tissue.

The remodelling stage can be divided into a consolidation and a maturation phase. The cellular tissue becomes more fibrous and the collagen fibres start to align, increasing strength. At the end of the consolidation phase, at about 10-12 weeks, and with the beginning of the maturation phase, the fibrous tissue is converted to a stronger scar tissue. The tissue continues to remodel for up to 1 year.

During the healing process, several factors participate in tissue repair. Among them, nitric oxide (NO) was described to improve tissue healing in animal models and to enhance clinical recovery of tendinopathies in humans (Molloy et al., 2003).

Non-invasive pharmacological treatments using antiinflammatory medications and corticosteroids act to minimize the deleterious side-effects of inflammation and preserve tissue integrity by blocking the initial inflammatory process of tendon/ligament healing. Nevertheless, the first phase of repair is severely affected, which can limit tissue complete regeneration.

Appropriate nutrition is vital for rapid healing, minimization of adhesion and restoration of gliding. In tendons, nutrients are provided through the vascularization network in addition to synovial fluid diffusion, as some areas are avascular.

Several factors have been described to affect tendon healing, and adhesion formation, including some surgical techniques and postoperative motion. Postoperative scar formation, and adhesions formed between the tendon and its surroundings are the most common complication (Beredjiklian, 2003) and a major clinical challenge, as scar tissue and adhesions result in the loss of normal tendon gliding, loss of motion, contracture formation, and functional disability (Beredjiklian, 2003).

One of the major causes of adhesion is a tendon sheath defect after traumatic or surgical injury. The tendon sheath is a membrane-like structure, which acts like a biological barrier, preventing invasion of peripheral fibrotic tissue and inhibiting exogenous healing of tendon. When this barrier is damaged, the normal functionality of the tendon is jeopardized.

Adhesion formation is also increased after an injury, ischaemia, immobilization and gapping at the repair site. Such complications compromise tissue properties, interfering with motion, gliding and consequently functionality. Experimental attempts to minimize adhesion formation embrace oral administration of steroids, antihistamines or NSAIDS, topical application of hyaluronic acid, collagen solutions or fibrin, or even silicone/cellophane wrapping, polyethylene tubes, interposed sheath flaps.

Healing after injury is structure specific. As in tendons, site-specific structural, biochemical and cellular differences (Zhang et al., 2011b) exist in ligaments, due to different mechanical demands and the nutritional environment. Some ligaments, such as the medial collateral ligament (MCL), have good healing potential, whereas others, such as the ACL, have a poor chance of healing after total rupture. Recently, a study conducted by Zhang et al. (2011b) showed that adult stem cells from different ligament tissues may behave differently in repair and regeneration processes. Since adult stem cells play a major role in repairing injuries, it is likely that those dissimilarities may be related to the differential healing capacities of the damaged tissue.

\subsection{Current treatments}

Tendon and ligament injuries can dramatically affect a patient's quality of life, and are expected to increase in number and severity in both ageing and active population. Tendon injuries, whether acute or chronic, are commonly managed either conservatively or surgically. Conservative management, such as rest, corticosteroid injection, orthotics, ultrasound, laser treatment or shockwave (Riley, 2004; Stergioulas et al., 2008) provide pain relief but, when they fail, surgery is required. Surgical procedures repair the damaged tendon in acute injuries but, in the case of chronic tendinopathies, excision of the damaged area might be performed. Despite the effort to improve the quality of repaired tendons, when compared to healthy tendons, surgically repaired tendons have inferior functionalities. Clinical approaches frequently imply working with degenerative tendon/ligament tissues, with an increased risk of failure and recurrence into a second surgery. The loss of mechanical competence is mainly due to a distorted ECM composition and a misalignment of collagen fibrils in the scar tissue.

Besides the risks inherent to any surgery; additional considerable risks are associated with tendon repair, including scarring and fibrous adhesion formation at the wound site, nerve damage and infection. The failure to achieve proper joint biomechanics must also be considered, since a partial loss of function can also lead to other diseases, such as osteoarthritis (Fleming et al., 2005).

Ligament injuries include a complete tear, a partial tear, a stretch injury (plastic deformation) or an alteration in function caused by an adjacent fracture. Following a similar path to tendon approaches, studies of ligament healing have traditionally been focused on surgical management and rehabilitation programmes that include bracing and physical modalities crucial in regaining function. In some situations, exercise may also produce beneficial effects, since ligaments are sensitive to training and disuse. 
Similarly to tendon, the scar tissue formed in ligament healing is mechanically inferior to normal tissue. Physiotherapy and early joint mobilization can reduce adhesions in these tissues, enhance vascularity and facilitate collagen realignment and faster healing, resulting in better gliding and higher tensile strength, preventing subsequent postoperative joint stiffness.

\subsubsection{Grafts}

In severe damage, biological grafts may be needed to replace damaged tendons. Nevertheless, in most cases, grafts do not provide adequate mechanical strength during the remodelling process.

Autografts are a currently used methodology to repair the affected tendon, preventing instability and reducing the rate of rerupture. Autografts may also lead to high morbidity and functional disability at the donor site and limited availability. Tissue laxity, mechanical mismatch and poor tissue integration (with a possible necrosis after implantation) are also disadvantages to be considered.

Patellar tendon and ACL tissues are commonly used autografts for tendinopathies. Interestingly, the reconstruction of ACL usually involves harvesting autogenous patellar tendon or hamstring tendon grafts as ligament substitutes (Larson, 1996).

Allografts are also alternative therapies for tendon and ligament repair but are not ideal, due to the associated risk of disease transmission and tissue rejection.

\subsubsection{Artificial prostheses}

Tendon and ligament tissues are continuously subjected to mechanical loads, such as muscle contraction and body movements. This natural environment is often associated with mechanical failures that restrict the successful application of most of the prosthetic replacements as satisfactory long-term tendon or ligament substitutes.

Nevertheless, since the 1970s, several prosthetic devices have been proposed for ligament replacement. Some commercial products were available as ligament substitutes, including Gore-Tex ${ }^{\circledR}$ (polytetrafluoroethylene), Lars ${ }^{\circledR}$ ligament (terephthalic polyethylene polyester - approved in Europe and Canada but not in the USA) and Leeds-Keio ${ }^{\circledR}$ (polyester ethylene terephthalate) (Chen et al., 2009a). Although these products showed short-term satisfactory results, long-term studies are ambiguous and reveal many associated complications.

Despite the fact that ligament prostheses exhibit mechanical properties equal to or exceeding the properties of normal human tissue, prosthetics have traditionally tended to be inadequate, due to postsurgical complications arising from wear and degeneration (Mascarenhas and MacDonald, 2008).

The initial mechanical properties of the reconstructed bone-ligament-bone complex depend not only on the mechanical properties of the prosthesis but also on the method of surgical fixation to the bones, as prosthesis stiffness and modulus can be reduced due to the effects of surgical fixation (suture, staples, screws, washers or combinations of these methods). Additionally, a permanent synthetic prosthesis that does not receive host tissue ingrowth is prone to long-term mechanical failure in the joint.

Despite all the effort, to date, no prosthesis has proven itself as a viable alternative to autografts for primary ACL reconstruction.

\subsubsection{Graft-augmentation devices}

Graft augmentation devices were developed to provide immediate protection and share mechanical loads with the biological graft until revascularization is complete and the ingrowth tissue capable of withstanding local tensile and compressive forces (Mascarenhas and MacDonald, 2008). Since these devices are meant to be temporarily used, long-term maintenance of the mechanical properties of the device is not necessary or even desirable. Ideally, graft augmentation devices should be resorbable, gradually transferring mechanical loads completely to the biological graft. Dacron (polyethylene terephthalate) and Kennedy LAD (braided polypropylene yarn) have been clinically used as FDA-approved graft ligament augmentation devices (LADs). Dacron minimizes abrasion of the graft and acts as a scaffold for fibrous tissue ingrowth (Mascarenhas and MacDonald, 2008), while Kennedy LAD protects the graft from excessive stresses but reveals a weak implant-graft interface (Mascarenhas and MacDonald, 2008).

\section{Strategies for repair and regeneration: designing tendon and ligament tissue substitutes}

As described previously, currently used methodologies mainly enable replacement or minimization of the damage caused in the tissue, controlling the pain or replacing the pathological tissue. To date, no clinical long-standing acceptable tendon or ligament substitute is available.

Tissue engineering (TE) has offered great potential in the treatment of tendon/ligament injuries, seeking a biological replacement that results in a fully regenerated living autologous tissue, mimicking the natural structure and function and with long-term viability (Figure 1).

As dense and well-organized connective tissue, much of tendon/ligament function is attributed to its intrinsic structural features. These characteristics include the positioning of ECM collagen fibrils according to tensile stress, that together with high water content are responsible for their visco-elastic and plastic properties, hence ensuring their mechanical function and, ultimately, stabilizing the synovial joint. These remarkable properties challenge the design of biomaterials for modulating biological responses into full repair and regeneration of the injury, thus envisioning successful long-term clinical outcomes (Table 1). 
In many soft connective tissue approaches, the mechanical properties for the collagen-hierarchy reconstruction are influenced by fibre alignment. Fibre alignment is meant primarily to mimic the resident ECM in tendon and ligaments, although some studies report that fibre alignment can also improve the biological response of cells seeded onto aligned scaffolds (Gigante et al., 2009; Teh et al., 2011). In opposition, knitting (Chen et al., 2010; Fan et al., 2009; Teh et al., 2011) or braiding (Cooper et al., 2005; Van Eijk et al., 2007) techniques have been described to contribute more substantially to the mechanical robustness of the system, improving the ability to sustain and distribute the natural loading stress.

\subsection{Biological scaffolds}

Biological scaffolds consist of protein-based extracellular matrices that are mammalian-derived tissues from human, equine, porcine and bovine sources (revised by Badylak et al., 2009; Chen et al., 2009a). Tissues such as small intestine mucosa, pericardium or dermis are processed to remove non-collagen components, likely to cause rejection, while retaining the natural collagen structure (predominantly collagen I fibres) and mechanical properties (Chen et al., 2009a). Restore ${ }^{\mathrm{TM}}$ (porcine small intestine mucosa), OrthADAPT ${ }^{\mathrm{TM}}$ (equine
Table 1. Scaffold requirements aiming at tendon or ligament tissue engineering

\begin{tabular}{|c|c|}
\hline Scaffold properties & Requirements \\
\hline Biodegradability & $\begin{array}{l}\text { Degradation rate adjusted to tissue } \\
\text { regeneration and restore of function }\end{array}$ \\
\hline Biocompatibility & $\begin{array}{l}\text { Do not elicit an immunological response } \\
\text { Interaction with the surrounding tissues } \\
\text { Induce host tissue integration }\end{array}$ \\
\hline Processability & $\begin{array}{l}\text { Design different architectural structures } \\
\text { mimicking native tissue and addressing } \\
\text { tissue needs } \\
\text { Match tissue size and shape }\end{array}$ \\
\hline Mechanical strength & $\begin{array}{l}\text { Ensure scaffold integrity with constant } \\
\text { loading } \\
\text { Allow active range of free movements } \\
\text { Prevent frictionless movements } \\
\text { Allow smooth and efficient gliding }\end{array}$ \\
\hline Biofunctionality & $\begin{array}{l}\text { Stimulate local environment into } \\
\text { regeneration } \\
\text { Biomechanically stable } \\
\text { With potential to be vascularized and } \\
\text { promote nutrients diffusion } \\
\text { Avoid adhesion formation } \\
\text { Assist cell infiltration, proliferation and } \\
\text { differentiation (if required) until complete } \\
\text { healing } \\
\text { Assist the delivery of therapeutic molecules } \\
\text { in growth factor strategies }\end{array}$ \\
\hline Others & Easy to handle, store and sterilize \\
\hline
\end{tabular}

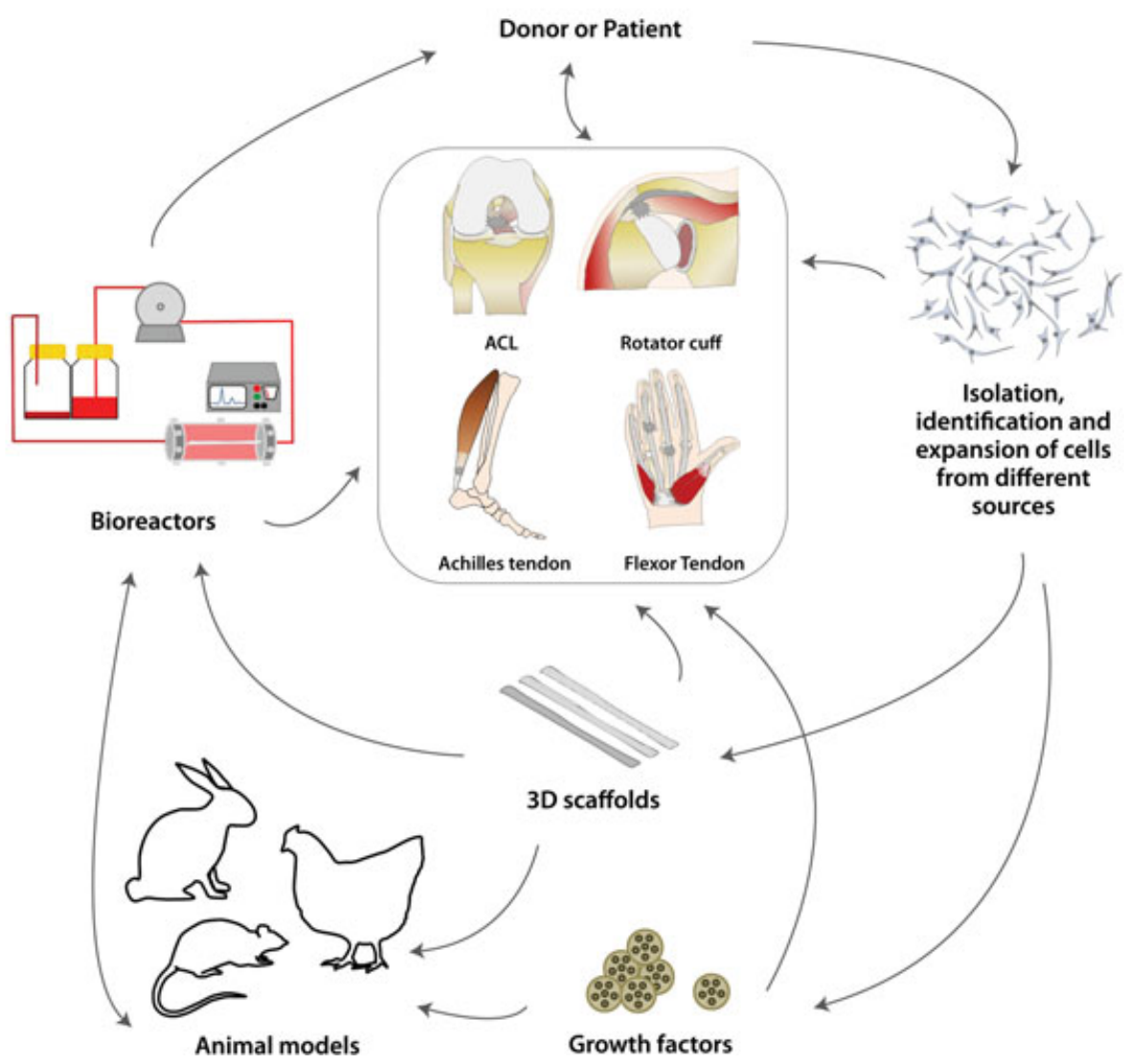

Figure 1. Schematic representation of tissue-engineered strategies for repair and regeneration of mostly injury-affected tendon and ligament tissues 
pericardium) or Bio-Blanket ${ }^{\circledR}$ (bovine dermis) are some of the FDA-approved biological scaffolds for ligament and tendon applications (Chen et al., 2009a). The major drawback of these commercial biological scaffolds is that their mechanical properties are significantly lower than those of normal tendons and ligaments (Chen et al., 2009a).

Naturally occurring scaffolds, such as small intestinal submucosa, have been clinically used for augmentation of the injured rotator cuff and Achilles tendon, and could be expanded to develop engineered substitutes for ACL (Brune et al., 2007).

Decellularized allograft tissues followed by recellularization in vitro are also another type of biological scaffold used in tendon (Omae et al., 2009) and ligament (Tischer et al., 2007) regenerative approaches. These scaffolds promise advantages over allografts, as they preserve the natural structure, biomechanical strength and stability (Tischer et al., 2007), and because of their reduced immunogenicity. Since native ECM matrices confer a unique environment, matching tendon/ligament needs in terms of cell attachment, proliferation, migration and gas and nutrient/metabolite diffusion, they have themselves been considered as scaffolds (Little et al., 2010; Zhang et al., 2011a). Nevertheless, the efficiency of cell removal, always inferior to $100 \%$, varies accordingly to the decellularization method used, viz. enzymatic, chemical, physical or a combination of these. Whatever the method, significant drawbacks must be considered, as enzymatic methods include animal-derived enzymes that can potentially invoke an adverse immune response by the host, and keeping in mind that the residual chemicals left after cell removal are likely to be toxic to host cells when the scaffold is implanted in vivo. Physical procedures may also partially disrupt structural and functional components of the ECM, possibly affecting the mechanical behaviour, degradation rate and bioactivity of the biological scaffold (Gilbert et al., 2006).

\subsection{Synthetic scaffolds}

Unlike biological scaffolds, synthetic commercial scaffolds in graft-augmentation devices and artificial prostheses can have much stronger mechanical properties than ligament and tendon ones, but their biocompatibility is limited (Chen et al., 2009a). In order to overcome this problem, alternative scaffolds have been tailored, following a TE strategy, for a more realistic application in tendon or ligament reconstruction. Synthetic polymers aimed at TE are typically more versatile than natural ones, enabling tailoring and controlling chemical and physical properties and structural features. They also represent a more reliable source of raw materials, and low immunogenicity potential (Tsuji and Ishizaka, 2001).

Poly- $\alpha$-hydroxyesters, such as polyglycolic acid (PGA) (Liu et al., 2006; Stoll et al., 2011), polylactic acid (PLA) and co-polymers (Cooper et al., 2005; Jenner et al., 2007), have been widely used in orthopaedic TE, including in tendon/ligament strategies. Although results from these studies were promising, scaffolds made of PGA, for instance, have limited application due to their mechanical brittleness and the lack of functional groups for signalling molecules. The manipulation of structural parameters in the design of scaffolds and their bioactivation, through the incorporation of soluble and insoluble signals for promoting cell activities, is likely to improve the neoformation of tissues. Materials such as oligo[poly (ethylene glycol) fumarate] (OPF) can be tailored to present covalently incorporated bioactive moieties. This feature has been explored in a hydrogel matrix as a cell carrier system for tendon and ligament regeneration (Doroski et al., 2010).

\subsection{Natural-based scaffolds}

A few scaffolds based on natural polymers have been proposed for tendon regeneration. For example, silk-based materials have shown great potential in ligament strategies (Fan et al., 2009; Moreau et al., 2005; Teh et al., 2011). Silk is a degradable and biocompatible material with intrinsic mechanical features. To investigate the ACL regeneration in vivo, Fan et al. (2009) developed a scaffold by rolling a microporous fibre silk mesh around braided silk cord to increase its mechanical properties, evidencing promising results for clinical applications.

Collagen is also a favourite material (Friess, 1998), since collagen is the major ECM component in tendons and ligaments. Several studies have demonstrated the applicability of collagen gels (Haddad-Weber et al., 2010) and multilamellar membranes (Gigante et al., 2009) mediating tendon repair, including in animal models (Awad et al., 1999). Nevertheless, several disadvantages of collagen-based scaffolds should be considered when aiming at a tendon/ligament substitute (Friess, 1998), including cost, variations from batch to batch and the possibility of immunogenic and disease transmission risks (Friess, 1998).

Fibrin is another natural biomaterial that has been proposed as a matrix for tendon and ligament strategies (Bayer et al., 2010; Hankemeier et al., 2009). Fibrin is a fibrous protein naturally involved in the clotting of blood, which polymerizes to form a haemostatic plug or clot over a wound site. Additionally, fibrin is a biodegradable and bioresorbable structure that can be used to encapsulate cells in a stable way (Hankemeier et al., 2009), thus acting as a supportive gel for assisting collagen fibrillonogenesis (Bayer et al., 2010) or through controlled delivery of growth factors stimulating the biological response towards tendon/ligament repair (Thomopoulos et al., 2007).

The combination of different biomaterials is also a strategic design for achieving hybrid scaffolds (Chen et al., 2010). Silk-collagen hybrid scaffolds developed in a knitted sponge matrix have been described, as well as the application of alginate and chitosan hybrid fibres to support tendon fibroblast adhesion (Shimode et al., 2009). 
Other polymers, including polysaccharides and proteins that share similar features with the natural ECM (revised by Mano et al., 2007), have shown interesting properties and outcomes in several tissues (Silva et al., 2010) and may also have a potential application in scaffold design for the tendon/ligament field.

\subsection{Cell sources for tendon/ligament}

The newborn tendon has a very high cell:matrix ratio that decreases during development, maturation and ageing, supporting the importance of the cellular contribution in tissue maintenance and functionality. The most widespread TE approach is to reconstruct a ligament/tendon by providing a scaffold seeded with cell-inducing neotissue formation that adequately meets the required biological and mechanical properties. The development of TE products with the application of appropriate types of cells could potentially improve the functionality and structure of tissue-engineered constructs. Cell-based therapies offer the potential to induce a regenerative response, rather than fibrous scarring, by stimulating local cells to proliferate and inspire the production of a structural matrix to ensure remodelling as the implant scaffold degrades.

Autologous cells are likely to provide an optimal approach to address a structural and functional replacement, avoiding immune reactions or morbidity caused by grafts. Tenocytes represent an obvious choice (Liu et al., 2006) that has been addressed in engineered strategies (Cooper et al., 2006; Gigante et al., 2009; Stoll et al., 2011). Tenocyte isolation and culture from different anatomical sites, such as at the rotator cuff (Pauly et al., 2010), patellar and Achilles tendons (Bernard-Beaubois et al., 1997; Bayer et al., 2010; Zhang and Wang, 2010), is feasible, but tenoblasts/tenocytes are scarce cells and thus increase tissue morbidity at the harvesting site, creating an undesirable side-defect.

Recent studies suggested the application of cells harvested from either intact (Brune et al., 2007; Cooper et al., 2005) or ruptured (Brune et al., 2007) ACL for TE approaches. Other studies mention harvesting patellar tendon samples during reconstruction of the ACL (Hankemeier et al., 2009). Despite the fact that fibroblasts obtained from damaged tissue could provide an interesting cell source, the availability of native cells might be limited and their functionality might be compromised, depending on the conditions and dimensions of the damaged tissue.

Dermis fibroblasts (DFs) are an easily accessible source of cells that has also been explored for tendon and ligament repair (Gigante et al., 2009; Van Eijk et al., 2007; Tischer et al., 2007), including for autologous approaches (Liu et al., 2006). DFs are commonly harvested from native tissue through a skin biopsy, which is a simple, timeless and cost-effective procedure and easily expanded during in vitro culture. More importantly, DFs share common characteristics with tenocytes, as both cell types are terminally differentiated cells and originated from mesoderm.
Also from an autologous source are cells obtained from the tendon sheath ( $\mathrm{Xu}$ et al., 2010), a membrane-like structure that involves tendons, separating tendon from the surrounding tissue and assuring tendon gliding. Nevertheless, disruption of the tendon sheath seriously interferes with normal tendon functionality, and consequently the isolation of these cells may severely compromise the healing approach, causing more damage than therapeutic outcomes.

The concept of using stem cells to accelerate repair and tissue regeneration has been established for various tissues, including cartilage or bone (revised by Rodrigues et al., 2011), showing its potential for future clinical outcomes. Considering the high self-renewal capacity and the ability to differentiate into several lineages, stem cells could improve the cellular density and proliferation rates at the tendon/ligament injuries. On account of the location and environmental milieu, resident stem cells from ligament and tendon tissues are an interesting source for tissue-regeneration approaches (Bi et al., 2007; Cheng et al., 2010; Lui and Chan, 2011; Murchison et al., 2007; Steinert et al., 2011; Zhang et al., 2011a). However, despite their potential, harvesting these cells might result in side-effects similar to those caused by tenoblast and ligament fibroblast procedures.

The synovial membrane that surrounds the posterior cruciate ligament would be a likely source of cells, especially for ACL. Synovial cells not only have a multi-lineage potential but also, in an untreated damage, these cells are likely to reach and colonize the injury site (Messenger et al., 2010).

Although it is very likely that all mesenchymal stem cells may become tendon-capable cells, stem cell-based strategies aiming at tendon and ligament studies have mainly approached bone marrow stem cells (BMSCs) (Awad et al., 1999; Cheng et al., 2010; Doroski et al., 2010; Fan et al., 2009; Hankemeier et al., 2009; Jenner et al., 2007; Moreau et al., 2005; Omae et al., 2009; Shimode et al., 2009; Teh et al., 2011). Interestingly, several comparative studies have been developed in the last few years, providing novel data concerning the search for the ideal cell source for tendon/ligament regeneration (Cheng et al., 2010; Cooper et al., 2006; Hankemeier et al., 2009; Van Eijk et al., 2007). For example, a comparative study between stem cells isolated from human ACL (LSCs) and bone marrow (BMSCs) from the same donors indicated that LSCs proliferated faster and maintained an undifferentiated state under basic fibroblast growth factor (bFGF) stimulation (Cheng et al., 2010). Moreover, when treated with transforming growth factor- $\beta 1$ (TGF $\beta 1$ ), LSCs upregulated major tendinous gene expression and produced a robust amount of ligament ECM protein (Cheng et al., 2010). When BMSCs were compared to mature fibroblasts encapsulated in a fibrin matrix, the results indicated that the molecular and biochemical parameters of tendon healing were enhanced in the presence of BMSCs (Hankemeier et al., 2009). The presence of BMSCs in window patellar tendon defect also mediates tendon repair outcomes, with a significant increment in 
maximum stress, modulus and strain energy density (Awad et al., 1999). Van Eijk et al. (2007) compared BMSCs, DFs and ACL cells with regard to in vitro proliferation and matrix production when seeded onto a PLGA multifilament scaffold. Preliminary results based on a single donor indicated that BMSCs were the most promising for application in ligament TE, followed by skin fibroblasts, whereas ACL fibroblasts seemed of limited application (Van Eijk et al., 2007). Concerning the parameters analysed in these comparative revisions, the stemness factor is likely to increase the cell potential in tendon/ligament TE.

The utilization of adipose tissue stem cells (ASCs) for tendon regeneration has recently been considered (Little et al., 2010; Park et al., 2010). ASCs present a high potential of application for $\mathrm{TE}$ and regenerative medicine approaches, as ASCs are easily differentiated into several lineages and the harvesting procedure causes minimal donor site morbidity and enables a large amount of tissue and cells to be obtained (Rada et al., 2009).

The most significant drawback using progenitor cells in vivo is the potential formation of ectopic bone and cartilage at the tendon/ligament site, due to their multipotential capacity and common origin (Cohen et al., 2010).

The wide-spectrum potential for differentiation makes fetal (Watts et al., 2011) or embryonic-derived stem cells (ESCs) (Chen et al., 2009b, 2010) another attractive cell source for healing and regenerative approaches. As with other SCs, ESCs application for tendon or ligament strategies has been barely explored (Chen et al., 2009b; Cohen et al., 2010), although evidence of success using connective tissue progenitor cells derived from ESCs has recently been reported in mouse Achilles tendon (Cohen et al., 2010).

Since no specific soluble factors to encourage tenogenesis have been identified, the study of tenogenesis in adults and tenogenic differentiation has been deterred by the lack of specific tendon markers.

Despite the different functions of connective tissue of cartilage, tendons, ligaments and fascia, these cells share a predominant origin from the embryonic mesodermal layer (Montero et al., 2011). Thus, the different phenotypic appearance is likely to be based on specific patterns of gene expression modulated by environmental factors. Several molecules associated to tendon/ligament formation and development are listed in Table 2, viz. TGF $\beta$ (Pryce et al., 2009), scleraxis (Murchison et al., 2007; Schweitzer et al., 2001; Shukunami et al., 2006) and several members of the small leucine-rich proteoglycans (SLRPs) (revised by (Banos et al., 2008), e.g. decorin and tenomodulin (Docheva et al., 2005; Shukunami et al., 2006). The natural presence of collagens I and III in tendon/ligament ECM has also been screened for normal tendon/ligament development approaches (Liu et al., 2011; Shukunami et al., 2006), and for the characterization of mature tendon/ ligament cells.

However, there are still clear limitations in defining optimal conditions for tenogenic differentiation and markers
Table 2. Molecules associated with tendon and ligament formation and development

\begin{tabular}{|c|c|}
\hline Transcription factors & Function in tendon/ligament tissues \\
\hline TGF- $\beta$ & $\begin{array}{l}\text { Inducer of tendon markers in mesenchymal } \\
\text { cells (Pryce et al., 2009) } \\
\text { Significant role in the genesis of tendons } \\
\text { and ligaments (Pryce et al., 2009) }\end{array}$ \\
\hline Scleraxis & $\begin{array}{l}\text { Considered a specific marker for tendon } \\
\text { progenitor cells } \\
\text { Associated to tendon differentiation and } \\
\text { ECM organization } \\
\text { Participates in the formation of force- } \\
\text { transmitting tendons (Schweitzer et al., } \\
2001 \text { ) } \\
\text { Highly expressed in tendon stem/progenitor } \\
\text { cells (TSPCs) (Murchison et al., 2007) }\end{array}$ \\
\hline Decorin (SLRPs) & $\begin{array}{l}\text { The most abundant PGs in tendon; binds to } \\
\text { TGF and EGF (Yoon and Halper, 2005) } \\
\text { Stabilizes and aligns collagen fibrils during } \\
\text { fibrinogenesis and contributes to tendon } \\
\text { strength and elasticity (revised by Banos } \\
\text { et al., 2008) }\end{array}$ \\
\hline Tenomodulin & $\begin{array}{l}\text { Regulator of tenocyte proliferation } \\
\text { Involved in collagen fibril maturation } \\
\text { (Docheva et al., 2005) } \\
\text { Highly expressed in tendon stem/progenitor } \\
\text { cells (Murchison et al., 2007) } \\
\text { Involved in the organization of TSPCs niche, } \\
\text { which in turn controls the fate of TSPCs (Bi } \\
\text { et al., 2007) }\end{array}$ \\
\hline Fibromodulin & $\begin{array}{l}\text { Binds to type I collagen } \\
\text { Facilitates formation of mature large } \\
\text { collagen fibrils } \\
\text { Involved in the modulation of tendon } \\
\text { strength (Yoon and Halper, 2005) }\end{array}$ \\
\hline Biglycan (SLRPs) & $\begin{array}{l}\text { Role in the regulation of collagen } \\
\text { fibrillogenesis during tendon development } \\
\text { (revised by Banos et al., 2008) } \\
\text { Involved in the organization of TSPCs niche, } \\
\text { which in turn controls the fate of TSPCs (Bi } \\
\text { et al., 2007) }\end{array}$ \\
\hline Collagen I & $\begin{array}{l}\text { The major collagen of tendons } \\
\text { Provides tensile stiffness (Gelse et al., 2003) }\end{array}$ \\
\hline Collagen III & $\begin{array}{l}\text { Structurally similar to collagen I; important } \\
\text { role in collagen I fibrillogenesis (revised by } \\
\text { Banos et al.,., 2008) } \\
\text { Widely distributed in collagen I-containing } \\
\text { tissues (Gelse et al., 2003) }\end{array}$ \\
\hline
\end{tabular}

to assess tenocyte/fibroblast phenotype. Genetically modified cells to produce and release higher amounts of the desired therapeutic factor are another option in cell-based strategies towards tendon/ligament regeneration. Preliminary studies have been conducted with TGF $\beta 1$ cDNA-transduced BMSCs grafts for acceleration and improvement of Achilles tendon healing in a rabbit model (Hou et al., 2009). In the ligamentogenic field, HaddadWeber et al. (2010) described the influence of transduced bone morphogenic protein-12 (BMP-12) and BMP-13 genes (also known as growth and differentiation factors GDF-5 and GDF-6, respectively) in the ligament differentiation process of BMSCs and ACL fibroblasts. After 21 days of cell culture within collagen I hydrogels, both cell types revealed a fibroblastic-like morphology. Nevertheless, BMSCs and ACLs differently expressed ligament-related markers, such as collagen III, decorin, scleraxis, tenascin and tenomodulin. 


\subsection{The role of growth factors in tendon/ ligament regeneration}

The tissue repair process is a complex cascade of biological events, orchestrated by numerous cytokines and growth factors. Repairs to tendon/ligament injuries are no exception. The delivery of humoral factors at the required dosages in a temporal and spatial pattern over the repair phase is critical to achieve a successful treatment. Scaffolds, microspheres and micro- or nanocapsules have been shown to be promising vehicles for growth factor delivery. Such technologies could provide crucial findings in regenerative medicine by controlling the spatiotemporal release of these molecules, ensuring long-term stability and storage of these factors in tailored systems. Despite the potential of these technologies, tendon/ligament tissues remain an unexplored field of interest.

Several growth factors are described to participate in tendon/ligament formation, ECM synthesis or healing, such as TGF $\beta$ (Pryce et al., 2009), insulin growth factor-1 (IGF-1; Olesen et al., 2006), platelet-derived growth factor (PDGF; Thomopoulos et al., 2007), bFGF (Cheng et al., 2010) or vascular endothelial growth factor (VEGF; Pufe et al., 2001). Other molecules, such as nitric oxide, have also been described to influence the volume of synthesized tissue during tendon healing (Molloy et al., 2003; Murrell et al., 1997) and to modulate gene expression and cellular adhesion of tenocytes (Molloy et al., 2003). When released at the site of injury, these growth factors modify cell proliferation, migration, differentiation and matrix synthesis, which could play a key role in stimulating local ECM production and local tenocytes (Liu et al., 2011). Table 3 summarizes growth factors that have been associated with the development of engineered tendon and ligament tissues. Sequential growth factor application in silk-BMSC engineered constructs has been proposed by Moreau et al. (2005). The idea was to assist BMSCs proliferation, providing FGF or epidermal growth factor (EGF) during the first days, and then to stimulate cell differentiation in the silk matrices for ECM production with TGF $\beta 1$ for an additional 9 days (Moreau et al., 2005). Although a significant increase in collagen type I transcript expression was observed in most conditions from day 5 of culture to day 14, mechanical integrity decreased in time, revealing the complexity of mechanisms and cell behaviour responses towards GF stimuli.

\subsubsection{Platelet-rich solutions}

Growing evidence on platelet-rich solutions suggests their application in tendon/ligament strategies (Anitua et al., 2005; Bosch et al., 2010; de Vos et al., 2010). Platelet-rich plasma (PRP) results from whole blood processing in order to obtain a high concentration of platelets. Platelets are a source of numerous growth factors which are released upon activation, including those involved in the repair and regeneration of several tissues. As an autologous biomaterial, PRP is immune- and transmissible
Table 3. Growth factors associated to engineered tendon and ligament tissues

Function in engineered tendon/ligament

\begin{tabular}{ll}
\hline TGF $\beta$ & TGF $\beta 1$ stimulates upregulation of gene \\
& expression and production of ECM in LSCs \\
& (Cheng et al., 2010). \\
& Associated with tendon formation, TGF $\beta$ \\
& signalling is a potent inducer of tendon markers \\
& in mesenchymal cells (Pryce et al., 2009). \\
& Increases hBMSCs proliferation in braided PLGA \\
& scaffolds (Jenner et al., 2007). \\
& Maintains undifferentiated state of LSCs and \\
& fastens cell proliferation (Cheng et al., 2010). \\
Involved in tendon graft remodelling (Petersen \\
bFGF \\
et al., 2003). \\
PEGF \\
Produced in vitro after plasma clots and platelets \\
stimuli by tendon cells (Anitua et al., 2005). \\
Controlled delivery of PDGF-BB enhanced the \\
biologic response of canine repaired flexor \\
tendons (Thomopoulos et al., 2007). \\
GDF-5 increases hBMSCs proliferation in braided \\
PLGA scaffolds (Jenner et al., 2007). \\
In ASCs, GDF-5 also led to increased proliferation \\
and enhanced ECM and tendonogenic markers \\
(Park et al., 2010). \\
GDF-5 deficiency in mice results in delayed \\
Achilles tendon repair (Chhabra et al., 2003). \\
Produced in vitro after plasma clots and platelets \\
stimuli by tendon cells (Anitua et al., 2005).
\end{tabular}

LSCs, ligament stem cells; HGF, hepatocyte growth factor.

disease-free, while combining the synergistic effect of multiple endogenous growth factors. Local injection of PRP has been associated with enhanced migration of circulationderived cells into the wound site at an early phase of the tendon healing process (Kajikawa et al., 2008). Anitua et al. (2005) also suggested that autologous protein and growth factors released from plasma clots and platelets act on human tendon cells, promoting proliferation and inducing the synthesis of angiogenic growth factors. Furthermore, PRP treatment could provide a less invasive alternative to surgery by promoting safe and natural healing. Nevertheless, pilot clinical trials on PRP therapies applied to tendinopathies in the Achilles tendon revealed inconclusive results (de Vos et al., 2010). Thus, understanding how PRP affects the healing processes of various musculoskeletal tissues could provide important cues in the factors and factor concentrations towards effective tendon/ligament regeneration.

\subsection{Bioreactors}

Tendons respond to mechanical forces by changing the metabolism as well as their structural and mechanical properties, which is commonly designated by tissue mechanical adaptation. Without the appropriate biomechanical cues, new tissue formation lacks the necessary collagenous organization and alignment for sufficient load-bearing capacity (Benhardt and Cosgriff-Hernandez, 2009). Mechanical loading of human tendon does result in a marked interstitial increase in growth factors that are known to stimulate synthesis of collagen and other 
ECM proteins (Kjaer et al., 2005). Therefore, bioreactors are an important tool in TE strategies, providing dynamic environments and mechanical stimulation to guide tissue remodelling and improve the performance of tendon/ligament tissue substitutes (Chen et al., 2010; Doroski et al., 2010; Messenger et al., 2010).

In fact, Chen et al. (2010) showed that dynamic mechanical stress directed ESCs, seeded onto a collagensilk scaffold, into a tenocyte-like morphology, demonstrated by the positive expression of tendon-related markers such as collagen I and scleraxis, as well as mechano-sensory organelles and molecules, namely cilia, myosin and integrins (Chen et al., 2010). Several studies have reported that mechanical conditioning positively affected cell proliferation and differentiation and increases tendon/ligament ECM synthesis (Doroski et al., 2010; Messenger et al., 2010). Cyclic strain significantly upregulated the collagen I, collagen III and tenascin-C genes of BMSCs after 21 days in culture, whereas genes for other pathways (osteogenic, chondrogenic and adipogenic) did not increase (Doroski et al., 2010). Additionally, cyclic tensile strain may also interfere in the alignment of collagen fibrils in the ECM. Preliminary studies indicate that, at a certain amplitude, ECM fibres aligned along the direction of the strain application, whereas in the control group the fibres were randomly oriented (Messenger et al., 2010).

\subsection{Animal models}

Despite the effort to solve tendon/ligament ruptures, few successful approaches have been achieved to completely regenerate these tissues. Animal models can greatly contribute to understanding the physiology and biomechanics of tendon/ligament repair mechanisms and provide the ultimate outcomes regarding the functionality of tissue engineered constructs.

For a better understanding about the mechanisms associated with ligament and tendon healing, as well as to assess the functionality of different regenerative therapies, several animal models have been studied, considering the type and degree of injury (Carpenter and Hankenson, 2004; Goh et al., 2003). Hens have been proposed as experimental models for flexon tendon studies and tendon sheath (Xu et al., 2010). Hen tendons are large enough for laceration and repair studies and are described to be an excellent model for studying the formation and prevention of adhesions. Conversely, the tendonsheath complex is an integrated structure, important for tendon function in hand and foot, where very efficient lubrication is required, due to intense mechanical stress (Sharma and Maffulli, 2005). Recently, an in vivo reconstruction of a tendon sheath was successful performed using tendon sheath-derived cells and polyglycolic acid (PGA) fibres in a Leghorn hen model ( $\mathrm{Xu}$ et al., 2010).

The ligament size of mice and rats and the constant remodelling of the musculoskeletal system during the lifetime of the animals can be major drawbacks to consider in the interpretation of functional and biological outcomes. Nevertheless, rats and mice represent encourageing species for genetic engineering manipulations, useful for elucidating basic biological processes, studying gene relationships and disease phenotypes, towards modelling human clinical conditions. These animals yield exciting new insights into the study of tendon/ligament injuries and associated diseases in biomedical research (Docheva et al., 2005; Kajikawa et al., 2008; Murrell et al., 1997; Zhang et al., 2011a), as well as mechanisms of tendon/ligament development and healing (Chhabra et al., 2003; Cohen et al., 2010; Hankemeier et al., 2009; Pryce et al., 2009). Some studies have investigated a rat patellar tendon model treated with ESCs (Chen et al., 2009b), showing in situ structural and mechanical improvement; furthermore, the cells secreted matrix and differentiated factors resulting in the stimulation of the endogenous regeneration process in tendon (Chen et al., 2009b).

The biochemical and functional properties of rabbit tendons have been well documented in biomedical research (Awad et al., 1999; Katsura et al., 2006; Stoll et al., 2011). However, the rabbit spends most of its lifetime in ventral recumbency with deeper knee flexion than in larger animals, such as goat, sheep or pig. Besides, rabbit posterior knees have stronger muscles and tendon/ligament structures, which may make it more difficult to mimic human tissue before and during regeneration. Studies on tendon properties (Dressler et al., 2002; Katsura et al., 2006) and patellar (Awad et al., 1999) and Achilles tendon (Stoll et al., 2011) regeneration strategies have been evaluated in the lapine model.

Dogs have also been recognized in tendon/ligament experimental models (Thomopoulos et al., 2007) but are not so frequently used, due to the proximity of dogs to humans as companion animals. Nevertheless, several studies focus on canine flexor tendons because of the similarity to those of humans (Thomopoulos et al., 2007).

The larger joint size in goat and sheep suggests that they are ideally suited for cruciate ligament grafting and reconstructions (Petersen et al., 2003). Other studies on the healing properties of adult and fetal tendons have also been considered in sheep models aiming at novel therapeutic strategies in the clinical set (Beredjiklian et al., 2003).

The degree of flexion in the stifle joints of goats and sheep suggests a closer analogy to human knee joints, despite dissimilarities in magnitude and direction of force in both the anteromedial and posterolateral bundles of ACL (Fan et al., 2009).

Pig anatomy also presents some limitations when compared to humans. For instance, the pig knee differs from that of the human in the magnitude and direction of force in the posterior lateral bundle of the ACL (Fan et al., 2009). Nevertheless, a study performed with BMSCs and a silk scaffold for ACL regeneration revealed that the regenerated ligament showed similarities to the native ACL, both in ECM composition and mechanical 
properties (Fan et al., 2009). Hybrid pigs are also a model for autologous approaches. Liu et al. (2006) tissueengineered autologous dermal fibroblasts and tenocytes with PGA unwoven fibre scaffolds as a strategy to repair a defect of the flexor digital superficial tendon. The results indicated that engineered tendons with both cell types exhibited similar macroscopic, histological and biomechanical features, with the neotissue histology showing a regular structure similar to that of normal tendon.

Horses are not a conventional and easily available model for human clinical outcomes, due to the demanding and expensive housing, handling and care of these animals. Nevertheless, horses represent a substantial value for the related sports and recreational activities, and hold great promise as a model for a wide range of medical conditions found in both horses and humans, predicting potential human outcomes. In the last few years, studies have been published describing various tendon-engineered strategies, including the development of equine induced pluripotent stem (iPS) cells (Nagy et al., 2011) and research on cell therapies. These approaches are not only promising for equine therapies but allow the validation of stem cell-based therapies in a large animal model, before moving into the human clinical scenario (Nagy et al., 2011). Another frequent study line is the use of horses for evaluating repair mechanisms of the superficial digital flexor tendon (Bosch et al., 2010; Watts et al., 2011). Watts et al. (2011) investigated the influence of intralesional injection of fetal-derived embryonic-like stem cells in the healing of tendonitis, verifying that tendon architecture and the lesion size were significantly improved in the fdESC-treated tendons.

\section{Conclusions and remarks on the future}

At the beginning of the twenty-first century, the study of tendon/ligament tissue regeneration is barely explored. Nevertheless, tendon/ligament injuries are quite frequent, affecting young and older patients, and result in considerable disability, which can aggravate into other pathologies, such as osteoarthritis. Since the working-age population is also affected, damage is also related to loss of working capability and health care costs.
The development of new strategies to stimulate and interact with the in vivo environment, enhancing the healing and functionality of tendon/ligament tissues, may yield more successful outcomes for clinical treatments than graft surgery replacements. A wide range of potential biomaterials are to be investigated, considering tendon/ligament features in general and the specific properties and needs of tendon/ligament in each anatomical region of interest.

The replacement of injured tissues requires cells to maintain and proliferate with the desired phenotype in order to achieve complete functionality. Since tenocyte harvesting is associated with severe disadvantages to the donor site, and might not be applicable in clinical cases; novel possibilities with stem cells are to be explored to better mimic the biophysical and biochemical milieu, enhancing ligament and tendon healing. The development of a specific expansion and differentiation medium could also accelerate the spreading of stem cell-based strategies for tendon/ligament.

Growing evidence on embryonic development can provide important clues towards promising developments on tissue-engineering products aiming at successful clinical methodologies in tendon/ligament tissues (Figure 1).

The importance of growth factor release in the healing process should also be considered, especially looking at growth factors that participate in tendon/ligament healing.

Considering that mechanical stimulus is an important niche for tendon/ligament development after birth and essential for a healthy and functional tissue, bioreactors may have a pivotal role in repair and regeneration of these tissues.

Future steps should include the optimization of the acquired knowledge and combination among the systems proposed, considering the advantages and disadvantages of each technique or procedure. Growing evidence on biological features in the body, including ECM biology, immunology and cell-cell and cell-scaffold interactions, will provide a better understanding of how the body responds to specific materials and will assist the scaffolding design of novel and functional structures that will mimic and fulfil the structural and biochemical needs of a desirable supportive device. A successful integration of scientific information will provide understanding and evolution of a tissue-engineered product aiming at practical tendon/ligament regeneration.

\section{References}

Anitua E, Andia I, Sanchez M et al. 2005; Autologous preparations rich in growth factors promote proliferation and induce VEGF and HGF production by human tendon cells in culture. J Orthop Res 23(2): 281-286.

Awad HA, Butler DL, Boivin GP et al. 1999; Autologous mesenchymal stem cell-mediated repair of tendon. Tissue Eng 5(3): 267-277.
Badylak SF, Freytes DO, Gilbert TW. 2009; Extracellular matrix as a biological scaffold material: structure and function. Acta Biomater 5(1): 1-13.

Banos CC, Thomas AH, Kuo CK. 2008; Collagen fibrillogenesis in tendon development: current models and regulation of fibril assembly. Birth Defects Res C Embryo Today 84(3): 228-244.
Bayer ML, Yeung CYC, Kadler KE et al. 2010; The initiation of embryonic-like collagen fibrillogenesis by adult human tendon fibroblasts when cultured under tension. Biomaterials 31(18): 4889-4897.

Benhardt HA, Cosgriff-Hernandez EM. 2009; The role of mechanical loading in ligament tissue engineering. Tissue Eng Part B Rev 15(4): 467-475. 
Beredjiklian PK. 2003; Biologic aspects of flexor tendon laceration and repair. $J$ Bone Joint Surg Am 85A(3): 539-550.

Beredjiklian PK, Favata M, Cartmell JS et al. 2003; Regenerative versus reparative healing in tendon: a study of biomechanical and histological properties in fetal sheep. Ann Biomed Eng 31(10): 1143-1152.

Bernard-Beaubois K, Hecquet C, Houcine O et al. 1997; Culture and characterization of juvenile rabbit tenocytes. Cell Biol Toxicol 13(2): 103-113.

Bi Y, Ehirchiou D, Kilts TM et al. 2007; Identification of tendon stem/progenitor cells and the role of the extracellular matrix in their niche. Nat Med 13(10): 1219-1227.

Bosch G, van Schie HT, de Groot MW et al. 2010; Effects of platelet-rich plasma on the quality of repair of mechanically induced core lesions in equine superficial digital flexor tendons: a placebocontrolled experimental study. J Orthop Res 28(2): 211-217.

Brune T, Borel A, Gilbert TW et al. 2007; In vitro comparison of human fibroblasts from intact and ruptured ACL for use in tissue engineering. Eur Cell Mater 14: 78-90; discussion, 90-71.

Carpenter JE, Hankenson KD. 2004; Animal models of tendon and ligament injuries for tissue engineering applications. Biomaterials 25(9): 1715-1722.

Carr AJ, Harvie P, Ostlere SJ et al. 2004; Genetic influences in the aetiology of tears of the rotator cuff - sibling risk of a full-thickness tear. $J$ Bone Joint Surg $B r$ 86B(5): 696-700.

Carroll CC, Dickinson JM, Haus JM et al. 2008; Influence of aging on the in vivo properties of human patellar tendon. $J$ Appl Physiol 105(6): 1907-1915.

Chen J, Xu J, Wang A et al. 2009a; Scaffolds for tendon and ligament repair: review of the efficacy of commercial products. Expert Rev Med Devices 6(1): 61-73.

Chen JL, Yin Z, Shen WL et al. 2010; Efficacy of hESC-MSCs in knitted silk-collagen scaffold for tendon tissue engineering and their roles. Biomaterials 31(36): 9438-9451.

Chen X, Song XH, Yin Z et al. 2009b; Stepwise differentiation of human embryonic stem cells promotes tendon regeneration by secreting fetal tendon matrix and differentiation factors. Stem Cells 27(6): 1276-1287.

Cheng MT, Liu CL, Chen TH et al. 2010; Comparison of potentials between stem cells isolated from human anterior cruciate ligament and bone marrow for ligament tissue engineering. Tissue Eng Part A 16(7): 2237-2253.

Chhabra A, Tsou D, Clark RT et al. 2003; GDF-5 deficiency in mice delays Achilles tendon healing. $J$ Orthop Res 21 (5): 826-835

Cohen S, Leshansky L, Zussman E et al. 2010; Repair of full-thickness tendon injury using connective tissue progenitors efficiently derived from human embryonic stem cells and fetal tissues. Tissue Eng Part A 16(10): 3119-3137.

Cooper JA Jr, Bailey LO, Carter JN et al. 2006; Evaluation of the anterior cruciate ligament, medial collateral ligament, Achilles tendon and patellar tendon as cell sources for tissue-engineered ligament. Biomaterials 27(13): 2747-2754.
Cooper JA, Lu HH, Ko FK et al. 2005; Fiberbased tissue-engineered scaffold for ligament replacement: design considerations and in vitro evaluation. Biomaterials 26 (13): 1523-1532.

Couppe C, Hansen P, Kongsgaard M et al. 2009; Mechanical properties and collagen cross-linking of the patellar tendon in old and young men. J Appl Physiol 107(3): 880-886.

Curwin SL. 2005; Rehabilitation after tendon injuries. In Tendon Injuries: Basic Science and Clinical Medicine, Maffulli N, Renström P, Leadbetter WB (eds). Springer-Verlag: London; 242-266.

de Vos RJ, Weir A, van Schie HT et al. 2010 Platelet-rich plasma injection for chronic Achilles tendinopathy: a randomized controlled trial. $J$ Am Med Assoc 303(2): 144-149.

Docheva D, Hunziker EB, Fassler $\mathrm{R}$ et al. 2005; Tenomodulin is necessary for tenocyte proliferation and tendon maturation. Mol Cell Biol 25(2): 699-705.

Doroski DM, Levenston ME, Temenoff JS 2010; Cyclic tensile culture promotes fibroblastic differentiation of marrow stromal cells encapsulated in poly(ethylene glycol)-based hydrogels. Tissue Eng Part A 16 (11): 3457-3466

Dressler MR, Butler DL, Wenstrup R et al. 2002; A potential mechanism for age-related declines in patellar tendon biomechanics. J Orthop Res 20(6): 1315-1322.

Fan H, Liu H, Toh SL et al. 2009; Anterior cruciate ligament regeneration using mesenchymal stem cells and silk scaffold in large animal model. Biomaterials 30(28): 4967-4977.

Fleming BC, Hulstyn MJ, Oksendahl HL et al. 2005; Ligament injury, reconstruction and osteoarthritis. Curr Opin Orthop 16(5): 354-362.

Frank CB. 2004; Ligament structure, physiology and function. $J$ Musculoskelet Neurona Interact 4(2): 199-201.

Friess W. 1998; Collagen - biomaterial for drug delivery. Eur J Pharm Biopharm 45 (2): 113-136.

Gelse K, Poschl E, Aigner T. 2003; Collagens - structure, function, and biosynthesis. Adv Drug Deliv Rev 55(12): 1531-1546.

Gigante A, Cesari E, Busilacchi A et al. 2009, Collagen I membranes for tendon repair: effect of collagen fiber orientation on cell behavior. J Orthop Res 27(6): 826-832.

Gilbert TW, Sellaro TL, Badylak SF. 2006 Decellularization of tissues and organs. Biomaterials 27(19): 3675-3683.

Goh JCH, Ouyang HW, Teoh SH et al. 2003; Tissue-engineering approach to the repair and regeneration of tendons and ligaments. Tissue Eng 9: S31-S44.

Gotlin RS, Huie G. 2000; Anterior cruciate ligament injuries. Operative and rehabilitative options. Phys Med Rehabil Clin N Am 11(4): 895-928.

Haddad-Weber M, Prager P, Kunz M et al. 2010; BMP12 and BMP13 gene transfer induce ligamentogenic differentiation in mesenchymal progenitor and anterior cruciate ligament cells. Cytotherapy 12(4): 505-513.

Hankemeier S, Hurschler C, Zeichen J et al. 2009; Bone marrow stromal cells in a liquid fibrin matrix improve the healing process of patellar tendon window defects. Tissue Eng Part A 15(5): 1019-1030.
Hou Y, Mao Z, Wei X et al. 2009; Effects of transforming growth factor- $\beta 1$ and vascular endothelial growth factor 165 gene transfer on Achilles tendon healing. Matrix Biol 28(6): 324-335.

Jenner JM, van Eijk F, Saris DB et al. 2007; Effect of transforming growth factor- $\beta$ and growth differentiation factor-5 on proliferation and matrix production by human bone marrow stromal cells cultured on braided poly lactic-co-glycolic acid scaffolds for ligament tissue engineering. Tissue Eng 13(7): 1573-1582.

Kajikawa Y, Morihara T, Sakamoto $\mathrm{H}$ et al. 2008; Platelet-rich plasma enhances the initial mobilization of circulation-derived cells for tendon healing. J Cell Physiol 215 (3): 837-845.

Katsura T, Tohyama H, Kondo E et al. 2006; Effects of administration of transforming growth factor (TGF)- $\beta 1$ and anti-TGF- $\beta 1$ antibody on the mechanical properties of the stress-shielded patellar tendon. $J$ Biomech 39(14): 2566-2572.

Khan RJ, Fick D, Keogh A et al. 2005; Treatment of acute achilles tendon ruptures. A meta-analysis of randomized, controlled trials. J Bone Joint Surg Am 87(10): 2202-2210.

Kjaer M, Langberg H, Miller BF et al. 2005; Metabolic activity and collagen turnover in human tendon in response to physical activity. $J$ Musculoskelet Neuronal Interact 5(1): 41-52.

Larson RV. 1996; Anterior cruciate ligament reconstruction with hamstring tendon, Operat Techn Orthop 6(3): 138-146.

Little D, Guilak F, Ruch DS. 2010; Ligamentderived matrix stimulates a ligamentous phenotype in human adipose-derived stem cells. Tissue Eng Part A 16(7): 2307-2319.

Liu CF, Aschbacher-Smith L, Barthelery NJ et al. 2011; What we should know before using tissue engineering techniques to repair injured tendons: a developmental biology perspective. Tissue Eng Part B Rev 17(3): 165-176.

Liu W, Chen B, Deng D et al. 2006; Repair of tendon defect with dermal fibroblast engineered tendon in a porcine model. Tissue Eng 12(4): 775-788.

Lui PP, Chan KM. 2011; Tendon-derived stem cells (TDSCs): from basic science to potential roles in tendon pathology and tissue engineering applications. Stem Cell Rev Rep 7(4): 883-897. DOI: 10.1007/s12015011-9276-0.

Maffulli N, Wong J, Almekinders LC. 2003; Types and epidemiology of tendinopathy. Clin Sports Med 22(4): 675-692.

Majewski M, Susanne H, Klaus S. 2006; Epidemiology of athletic knee injuries: a 10year study. Knee 13(3): 184-188.

Mano JF, Silva GA, Azevedo HS et al. 2007; Natural origin biodegradable systems in tissue engineering and regenerative medicine: present status and some moving trends. $J R$ Soc Interface 4(17): 999-1030.

Mascarenhas R, MacDonald PB. 2008; Anterior cruciate ligament reconstruction: a look at prosthetics - past, present and possible future. Mcoill J Med 11(1): 29-37.

Messenger MP, Raifel M, Seedhom BB et al. 2010; Enamel matrix derivative enhances tissue formation around scaffolds used for tissue engineering of ligaments. $J$ Tissue Eng Regen Med 4(2): 96-104. 
Molloy T, Wang Y, Murrell G. 2003; The roles of growth factors in tendon and ligament healing. Sports Med 33(5): 381-394.

Montero JA, Lorda-Diez CI, Hurle JM. 2011; Regenerative medicine and connective tissues: cartilage versus tendon. $J$ Tissue Eng Regen Med. DOI: 10.1002/term.436.

Moreau JE, Chen J, Horan RL et al. 2005; Sequential growth factor application in bone marrow stromal cell ligament engineering. Tissue Eng 11(11-12): 1887-1897.

Murchison ND, Price BA, Conner DA et al. 2007; Regulation of tendon differentiation by scleraxis distinguishes force-transmitting tendons from muscle-anchoring tendons. Development 134(14): 2697-2708.

Murrell GA, Szabo C, Hannafin JA et al. 1997; Modulation of tendon healing by nitric oxide. Inflamm Res 46(1): 19-27.

Nagy K, Sung HK, Zhang P et al. 2011; Induced pluripotent stem cell lines derived from equine fibroblasts. Stem Cell Rev 7 (3): 693-702.

Ng GYF. 2002; Ligament injury and repair: current concepts. Hong Kong Physiother $J$ 20(1): 22-29.

Olesen JL, Heinemeier KM, Haddad F et al. 2006; Expression of insulin-like growth factor I, insulin-like growth factor binding proteins, and collagen mRNA in mechanically loaded plantaris tendon. J Appl Physiol 101(1): 183-188.

Omae H, Zhao C, Sun YL et al. 2009; Multilayer tendon slices seeded with bone marrow stromal cells: a novel composite for tendon engineering. J Orthop Res 27(7): 937-942.

Park A, Hogan MV, Kesturu GS et al. 2010; Adipose-derived mesenchymal stem cells treated with growth differentiation factor5 express tendon-specific markers. Tissue Eng Part A 16(9): 2941-2951.

Pauly S, Klatte F, Strobel C et al. 2010; Characterization of tendon cell cultures of the human rotator cuff. Eur Cell Mater 20: 84-97.

Petersen W, Unterhauser F, Pufe $\mathrm{T}$ et al. 2003; The angiogenic peptide vascular endothelial growth factor (VEGF) is expressed during the remodeling of free tendon grafts in sheep. Arch Orthop Trauma Surg 123(4): 168-174.

Pryce BA, Watson SS, Murchison ND et al. 2009; Recruitment and maintenance of tendon progenitors by TGF $\beta$ signaling are essential for tendon formation. Development 136(8): 1351-1361.
Pufe T, Petersen W, Tillmann B et al. 2001; The angiogenic peptide vascular endothelial growth factor is expressed in fetal and ruptured tendons. Virchows Arch 439(4): 579-585.

Rada T, Reis RL, Gomes ME. 2009; Adipose tissue-derived stem cells and their application in bone and cartilage tissue engineering. Tissue Eng Part B Rev 15(2): 113-125. Reeves ND. 2006; Adaptation of the tendon to mechanical usage, $J$ Musculoskelet Neuronal Interact 6(2): 174-180.

Riley G. 2004; The pathogenesis of tendinopathy. A molecular perspective. Rheumatology (Oxf) 43(2): 131-142.

Rodrigues MT, Gomes ME, Reis RL. 2011; Current strategies for osteochondral regeneration: from stem cells to preclinical approaches. Curr Opin Biotechnol 22: 1-8.

Schweitzer R, Chyung JH, Murtaugh LC et al. 2001; Analysis of the tendon cell fate using Scleraxis, a specific marker for tendons and ligaments. Development 128(19): 3855-3866.

Sharma P, Maffulli N. 2005; Basic biology of tendon injury and healing. Surgeon 3(5): 309-316.

Sharma P, Maffulli N. 2006; Biology of tendon injury: healing, modeling and remodeling. J Musculoskelet Neuronal Interact 6 (2): 181-190.

Shimode K, Iwasaki N, Majima T et al. 2009; Local upregulation of stromal cell-derived factor-1 after ligament injuries enhances homing rate of bone marrow stromal cells in rats. Tissue Eng Part A 15(8): 2277-2284.

Shukunami C, Takimoto A, Oro $\mathrm{M}$ et al. 2006; Scleraxis positively regulates the expression of tenomodulin, a differentiation marker of tenocytes. Dev Biol 298 (1): 234-247.

Silva SS, Mano JF, Reis RL. 2010; Potential applications of natural origin polymerbased systems in soft tissue regeneration. Crit Rev Biotechnol 30(3): 200-221.

Steinert AF, Kunz M, Prager P et al. 2011; Mesenchymal stem cell characteristics of human anterior cruciate ligament outgrowth cells. Tissue Eng Part A 17(9-10): 1375-1388.

Stergioulas A, Stergioula M, Aarskog R et al. 2008; Effects of low-level laser therapy and eccentric exercises in the treatment of recreational athletes with chronic Achilles tendinopathy. Am J Sports Med 36(5): 881-887.
Stoll C, John T, Conrad C et al. 2011; Healing parameters in a rabbit partial tendon defect following tenocyte/biomaterial implantation. Biomaterials 32(21): 4806-4815.

Teh TK, Toh SL, Goh JC. 2011; Aligned hybrid silk scaffold for enhanced differentiation of mesenchymal stem cells into ligament fibroblasts. Tissue Eng Part C Methods 17(6): 687-703.

Thomopoulos S, Zaegel M, Das R et al. 2007; PDGF-BB released in tendon repair using a novel delivery system promotes cell proliferation and collagen remodeling. $J$ Orthop Res 25(10): 1358-1368.

Tischer T, Vogt S, Aryee S et al. 2007; Tissue engineering of the anterior cruciate ligament: a new method using acellularized tendon allografts and autologous fibroblasts. Arch Orthop Trauma Surg 127(9): 735-741.

Tsuji H, Ishizaka T. 2001; Blends of aliphatic polyesters. VI. Lipase-catalyzed hydrolysis and visualized phase structure of biodegradable blends from poly( $\varepsilon$-caprolactone) and poly(L-lactide). Int $J$ Biol Macromol 29 (2): 83-89.

Van Eijk F, Saris DBF, Riesle J, et al. 2007; Tissue engineering of ligaments: a comparison of bone marrow stromal cells, anterior crutiate ligament and skin fibroblasts as cell source. Tissue Eng 10(5/6): 893-903.

Watts AE, Yeager AE, Kopyov OV et al. 2011; Fetal derived embryonic-like stem cells improve healing in a large animal flexor tendonitis model. Stem Cell Res Ther 2(1): 4.

$\mathrm{Xu} \mathrm{L}$, Cao D, Liu W et al. 2010; In vivo engineering of a functional tendon sheath in a hen model. Biomaterials 31(14): 3894-3902.

Yoon JH, Halper J. 2005; Tendon proteoglycans: biochemistry and function. $J$ Musculoskelet Neuronal Interact 5(1): 22-34.

Zhang J, Li B, Wang JH. 2011a; The role of engineered tendon matrix in the stemness of tendon stem cells in vitro and the promotion of tendon-like tissue formation in vivo. Biomaterials 32(29): 6972-6981.

Zhang J, Pan T, Im HJ et al. 2011b; Differential properties of human ACL and MCL stem cells may be responsible for their differential healing capacity. BMC Med 9: 68.

Zhang J, Wang JH. 2010; Characterization of differential properties of rabbit tendon stem cells and tenocytes. BMC Musculoskelet Disord 11: 10. 